Applied Economics

\title{
Persistence in the private debt-t -GDP ratio: evidence from 43 OECD countries
}

\section{Guglielmo Maria Caporale, Luis Alberiko Gil-Alana \& Maria Malmierca}

To cite this article: Guglielmo Maria Caporale, Luis Alberiko Gil-Alana \& Maria Malmierca (2021) Persistence in the private debt-t -GDP ratio: evidence from 43 OECD countries, Applied Economics, 53:43, 5018-5027, DOI: 10.1080/00036846.2021.1912700

To link to this article: https://doi.org/10.1080/00036846.2021.1912700

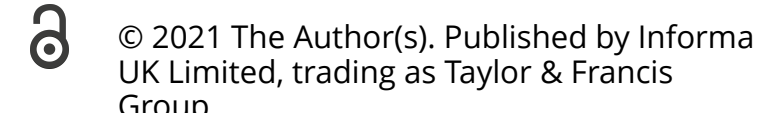
Group.

\section{曲 Published online: 07 Jun 2021.}

Submit your article to this journal $\sqsubset$

Џ Article views: 363

Q View related articles $\square$

View Crossmark data ¿ 


\title{
Persistence in the private debt-t -GDP ratio: evidence from 43 OECD countries
}

\author{
Guglielmo Maria Caporale $\mathbb{D D}^{\mathrm{a}}$, Luis Alberiko Gil-Alana $\mathbb{D}^{\mathrm{b}}$ and Maria Malmierca ${ }^{\mathrm{c}}$

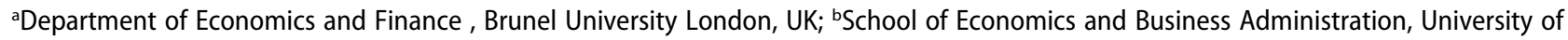 \\ Navarra, Pamplona, Spain; 'Faculty of Economics, University Villanueva, Madrid, Spain
}

ABSTRACT

This paper investigates the degree of persistence of the private debt-to-GDP ratio in 43 OECD countries by estimating the fractional integration parameter of each series. Almost all of them are found to be highly persistent, with orders of integration around or above 1. The only exception is Argentina, where the series appears to be mean-reverting. These results highlight the key importance of macroprudential policy as one of the pillars of macro policy.

\section{KEYWORDS}

Persistence; fractional integration; private debt; long memory

JEL CLASSIFICATION C22; G30; G51

\section{Introduction}

The 2007-8 global financial crisis (GFC) brought once again to the fore the importance of financial stability. One of the key aspects of the crisis was the fact that credit to the private sector was frozen, which led to a sharp fall in both private consumption and investment and thus in the growth rate of GDP. Having originated in the US as a subprime mortgage crisis, it quickly spread across the globe reducing lending and resulting in private sector deleveraging.

This paper investigates the statistical properties of the private debt-to-GDP ratio in 43 OECD countries with the aim of gaining a deeper understanding of its behaviour during the GFC. More specifically, the analysis uses a fractional integration approach to estimate the degree of persistence of the series of interest and to shed light on whether the effects of shocks hitting them are transitory or permanent. This type of framework is more general and flexible than the standard one based on the $\mathrm{I}(0)$ versus $\mathrm{I}(1)$ dichotomy since it allows not only for integer degrees of integration but also for fractional ones, and thus it considers a much wider range of stochastic processes. To our knowledge, no previous study has applied such methods in the case of the private debt-to-GDP ratio (Ramalho and Silva 2009, focused on firms only).
The results are informative about cross-country differences possibly accounting for the different impact of the GFC and can also be used for developing an appropriate macroprudential framework for safeguarding the stability of the financial system. This should involve monitoring private debt indicators to avoid excessive borrowing and policies, such as countercyclical capital requirements or dynamic provisions to boost private debt and encourage private investment and consumption during recessions.

The layout of the paper is as follows. Section 2 briefly reviews the relevant literature. Section 3 outlines the methodology. Section 4 presents the data and the empirical findings. Section 5 summarizes the main findings and discusses their policy implications.

\section{Literature review}

Numerous studies have concluded that private debt plays a crucial role in business cycle dynamics see, for instance, Kiyotaki and Moore (2002), Koo (2008), Raberto, Teglio, and Cincotti (2012) and Chen et al. (2015) among others. There is also an extensive literature focusing more specifically on the private deleveraging process that followed the GFC and its effects on the GDP path. Estrada et al. (2014) presented cross-country evidence of the relevance of the level of private debt for the slow

CONTACT Guglielmo Maria Caporale GuglielmoMaria.Caporale@brunel.ac.uk $\Theta$ Department of Economics and Finance, Brunel University London, Uxbridge UB8 3PH, UK.

Authors' alphabetical order has been applied in this paper.

(c) 2021 The Author(s). Published by Informa UK Limited, trading as Taylor \& Francis Group.

This is an Open Access article distributed under the terms of the Creative Commons Attribution License (http://creativecommons.org/licenses/by/4.0/), which permits unrestricted use, distribution, and reproduction in any medium, provided the original work is properly cited. 
recovery of consumption after 2008. Andrés et al. (2020), using a general equilibrium model, found that there is a relation between the size and speed of fiscal consolidations and the duration of private deleveraging; they argued that, after a negative shock to the economy, fiscal tightening results in private deleveraging and lower economic growth.

Eggertsson and Krugman (2012) developed a new Keynesian model to show that the negative effects of deleveraging on spending and output might be only temporary, which should be taken into account by policy-makers. They also showed that the level of debt initially held by households is crucial in terms of the effects of deleveraging. Ivens (2018) also analysed the role of policymakers in counteracting the welfare losses caused by a deleveraging shock and the issue of the optimal fiscal policy response to a private debt crisis.

Another strand of the literature examines macroprudential tools to stabilize private leverage (Quint and Rabanal 2014; Brzoza-Brzezina, Kolasa and Makarski, 2015; Rubio and Carrasco-Gallego 2014). In particular, De Blas and Malmierca (2020), Bole, Prašnikar, and Trobec (2014) and Dehmej and Gambacorta (2017) all argued that, after financial shocks, such as the GFC, the traditional monetary-fiscal policy mix needs to be complemented by macroprudential policies aimed at stabilizing both private and public debt.

Finally, Ramalho and Silva (2009) examined the determinants for the financial leverage decisions of firms using a fractional regression model and found that different factors determine whether or not debt is issued and the amount issued, respectively.

\section{Methodology}

This section outlines the fractional integration framework used for the analysis. A series is said to be fractionally integrated or integrated of order d, i.e. $\mathrm{I}(\mathrm{d})$, if it can be represented as:

$$
(1-B)^{d} x(t)=u(t), \quad t=1,2, \ldots,
$$

where $B$ is the backshift operator, i.e. $B^{k} x(t)=x$ $(t-k), u(t)$ is $I(0)$ or short memory (either a white noise or weakly autocorrelated as in the stationary ARMA processes) and where $\mathrm{d}$ can be any real value. Earlier studies assumed that $\mathrm{d}$ is equal to either 0 (stationarity) or 1 (nonstationarity) and carried out unit root tests to distinguish between these two cases (Dickey and Fuller 1979; Phillips and Perron 1988; Kwiatkowski et al. 1992; Elliot, Rothenberg, and Stock; Ng and Perron 2001; etc.). However, the differencing parameter $\mathrm{d}$ can also be a fractional value in the interval $(0,1)$ or even above 1 . In such a case, the polynomial in B in (1) can be expressed in terms of a Binomial expansion such that, for all real $d$ :

$$
\begin{aligned}
(1-B)^{d}= & \sum_{j=1}^{\infty}\left(\begin{array}{l}
d \\
j
\end{array}\right)(-1)^{j} B^{j} \\
= & 1-d B+\frac{d(d-1)}{2} B^{2} \\
& -\frac{d(d-1)(d-2)}{6} B^{3}+\ldots
\end{aligned}
$$

The higher the value of $d$ is, the higher is the degree of dependence between the observations; $d$ is also a measure of persistence. The following cases can be considered:

(1) short-memory processes, if $d=0$,

(2) long-memory stationary processes, if $0<\mathrm{d}<0.5$,

(3) nonstationary mean-reverting processes $(0.5 \leq \mathrm{d}<1)$

(4) unit roots or $\mathrm{I}(1)$ processes, if $\mathrm{d}=1$, and

(5) explosive patterns, if $d>1$.

Exogenous shocks to the series will have transitory effects as long as $\mathrm{d}$ is strictly below 1, whilst those effects will be permanent if $d \geq 1$, lower values of $\mathrm{d}$ corresponding to a faster meanreversion process.

We estimate $\mathrm{d}$ by using a frequency domain version of the Whittle functions as expressed in Dahlhaus (1989), implementing a simple version of the testing approach developed in Robinson (1994) which is valid even in non-stationary contexts $(\mathrm{d} \geq 0.5)$. This method is asymptotically normally distributed but also performs well in small samples (Gil-Alana, 2000; for its functional form, see, for example, Gil-Alana and Robinson 1997). 


\section{Data and empirical results}

We use quarterly data on credit to the private non-financial sector for 43 OECD countries for the period 1951-2020. The data source is the Bank of International Settlements (BIS) Statistics Warehouse. The series used are reported at market values. The 43 OECD countries considered are the following: Turkey, Malaysia, China, Hong Kong, Spain, Australia, Russia, Belgium, Italy, Chile, India, Austria, Saudi Arabia, Hungary, Japan, Norway, United States, Netherlands, Thailand, Canada, Korea, Argentina, New Zealand, Ireland, Singapore, France, Indonesia, Sweden, Luxembourg, Poland, Denmark, Israel, Brazil, Switzerland, Colombia, Mexico, Finland, Greece, Czech Republic, Portugal, United Kingdom, South Africa and Germany.

Table 1 specifies the sample period for each country. More than 100 observations are available in most cases, the only exceptions being Brazil (97 observations), Colombia (94 observations) and Luxembourg (85 observations).

Table 2 reports some descriptive statistics. In 24 countries the mean is above $100 \%$. It is noteworthy that this group of highly indebted countries includes none from Latin America; China, Hong Kong, Korea, Singapore and Japan are the only Asian economies with such debt levels; most of the European, North American and Oceanian countries exceed the $100 \%$ mark.

The estimated model is as follows:

$$
\begin{aligned}
y(t) & =\alpha+\beta t+x(t) ; \quad(1-B)^{d} x(t) \\
& =u(t), \quad t=1,2, \ldots
\end{aligned}
$$

where $y(t)$ is the observed time series, $\alpha$ and $\beta$ are unknown coefficients on the intercept and the linear time trend, and $\mathrm{x}(\mathrm{t})$ is assumed to be $\mathrm{I}(\mathrm{d})$, with $\mathrm{d}$ being another parameter to be estimated. Tables 3 and 4 report the estimates of $d$ (as well as the 95\% confidence bands of the non-rejection values of d using Robinson's (1994) test) for the two cases of white noise and autocorrelated errors respectively. In the latter case, the exponential spectral model of Bloomfield (1973) is adopted; this uses the spectral density function to log-approximate the log of the spectrum of an ARMA model; it displays autocorrelations also decaying exponen-

\begin{tabular}{|c|c|c|c|c|}
\hline Country & Abbrevation & Starting year & Ending year & N. of obs \\
\hline Argentina & $A R$ & $1984 Q 4$ & $2020 Q 1$ & 142 \\
\hline Austria & AT & $1960 \mathrm{Q} 4$ & $2020 \mathrm{Q} 1$ & 238 \\
\hline Australia & $A U$ & $1960 Q 2$ & $2020 Q 1$ & 240 \\
\hline Belgium & $\mathrm{BE}$ & $1970 Q 4$ & $2020 Q 1$ & 190 \\
\hline Brazil & $\mathrm{BR}$ & 199601 & 202001 & 97 \\
\hline Canada & CA & $1955 Q 4$ & $2020 Q 1$ & 258 \\
\hline Switzerland & $\mathrm{CH}$ & $1960 Q 4$ & $2020 Q 1$ & 238 \\
\hline Chile & $\mathrm{CL}$ & $1983 Q 1$ & $2020 Q 1$ & 149 \\
\hline China & $\mathrm{CN}$ & $1985 Q 4$ & $2020 Q 1$ & 138 \\
\hline Colombia & CO & 199604 & 202001 & 94 \\
\hline Czech Republic & $\mathrm{CZ}$ & $1993 Q 1$ & $2020 Q 1$ & 109 \\
\hline Germany & $\mathrm{DE}$ & $1960 Q 4$ & $2020 Q 1$ & 238 \\
\hline Denmark & DK & $1966 \mathrm{Q} 4$ & $2020 Q 1$ & 214 \\
\hline Spain & ES & 1970Q1 & $2020 \mathrm{Q} 1$ & 201 \\
\hline Finland & $\mathrm{FI}$ & $1970 Q 4$ & $2020 Q 1$ & 198 \\
\hline France & FR & $1969 Q 4$ & 202001 & 202 \\
\hline United Kingdom & GB & 196301 & 202001 & 229 \\
\hline Greece & $\mathrm{GR}$ & $1970 Q 4$ & $2020 \mathrm{Q} 1$ & 198 \\
\hline Hong Kong & HK & $1978 Q 4$ & $2020 Q 1$ & 166 \\
\hline Hungary & HU & $1970 Q 4$ & $2020 Q 1$ & 198 \\
\hline Indonesia & ID & 1976Q1 & $2020 Q 1$ & 177 \\
\hline Ireland & IE & 197102 & 202001 & 196 \\
\hline Israel & IL & $1990 \mathrm{Q}$ & $2020 Q 1$ & 118 \\
\hline India & IN & 1951Q2 & 2020Q1 & 276 \\
\hline Italy & IT & $1960 Q 4$ & 202001 & 238 \\
\hline Japan & $J P$ & $1964 Q 4$ & $2020 Q 1$ & 222 \\
\hline Korea & $\mathrm{KR}$ & $1962 Q 4$ & 202001 & 230 \\
\hline Luxembourg & LU & 199901 & $2020 Q 1$ & 85 \\
\hline Mexico & $M X$ & 1980Q4 & $2020 Q 1$ & 158 \\
\hline Malasya & MY & $1964 Q 2$ & $2020 Q 1$ & 224 \\
\hline Netherlands & $\mathrm{NL}$ & 1961Q1 & $2020 Q 1$ & 237 \\
\hline Norway & NO & 196004 & 202001 & 238 \\
\hline New Zeland & NZ & $1960 Q 4$ & $2020 \mathrm{Q} 1$ & 238 \\
\hline Poland & $\mathrm{PL}$ & 1992Q1 & $2020 Q 1$ & 113 \\
\hline Portugal & PT & $1960 Q 4$ & $2020 Q 1$ & 238 \\
\hline Russia & RU & 1995Q2 & $2020 Q 1$ & 100 \\
\hline Saudi Arabia & SA & 199301 & $2020 Q 1$ & 109 \\
\hline Sweden & SE & 1961Q1 & $2020 Q 1$ & 237 \\
\hline Singapore & SG & $1970 Q 4$ & $2020 Q 1$ & 198 \\
\hline Thailand & TH & $1970 Q 4$ & $2020 \mathrm{Q} 1$ & 198 \\
\hline Turkey & TR & 1986Q1 & $2020 Q 1$ & 137 \\
\hline United States & US & $1952 Q 1$ & $2020 Q 1$ & 273 \\
\hline South Africa & $Z A$ & 1965Q1 & $2020 \mathrm{Q} 1$ & 221 \\
\hline
\end{tabular}

Table 1. Set of countries and sample periods.

tially but is stationary for all range of parameters unlike the AR case.

Table 3 reports the results with white noise errors for three model specifications: i) no deterministic terms, ii) an intercept, and iii) an intercept and a linear time trend. We follow a general to specific approach, starting with the specification including both regressors and sequentially dropping any insignificant coefficients to select the best specification. The time trend is found to be significant in 13 out of the 43 countries examined, namely Austria, Belgium, Canada, Switzerland, 
Table 2. Descriptive statistics.

\begin{tabular}{|c|c|c|c|c|}
\hline Country & Mean & Std. Dev. & Max. value & Min. Value \\
\hline AR & 29.5 & 11.8 & 91.1 & 9.4 \\
\hline AT & 94.9 & 38.2 & 147.6 & 29.9 \\
\hline $\mathrm{AU}$ & 115.9 & 49.6 & 202.3 & 53.0 \\
\hline $\mathrm{BE}$ & 131.2 & 50.5 & 229.7 & 77.3 \\
\hline$B R$ & 57.1 & 10.6 & 78.3 & 44.0 \\
\hline$C A$ & 128.8 & 40.6 & 220.6 & 71.7 \\
\hline $\mathrm{CH}$ & 173.2 & 40.2 & 257.5 & 114.2 \\
\hline $\mathrm{CL}$ & 95.2 & 28.5 & 162.3 & 52.9 \\
\hline $\mathrm{CN}$ & 120.5 & 44.7 & 216.2 & 64.2 \\
\hline $\mathrm{CO}$ & 51.1 & 8.0 & 65.6 & 39.2 \\
\hline$C Z$ & 79.6 & 10.8 & 93.1 & 59.1 \\
\hline $\mathrm{DE}$ & 101.5 & 18.7 & 132.6 & 58.5 \\
\hline DK & 161.2 & 48.5 & 254.6 & 103.7 \\
\hline ES & 117.6 & 53.6 & 226.8 & 67.0 \\
\hline $\mathrm{FI}$ & 125.9 & 34.8 & 193.4 & 79.9 \\
\hline $\mathrm{FR}$ & 137.9 & 32.5 & 218.0 & 93.8 \\
\hline GB & 111.6 & 48.4 & 193.8 & 52.7 \\
\hline GR & 66.6 & 35.0 & 133.6 & 34.3 \\
\hline HK & 177.8 & 54.2 & 321.8 & 83.8 \\
\hline $\mathrm{HU}$ & 70.3 & 25.7 & 136.9 & 35.4 \\
\hline ID & 36.3 & 16.0 & 129.5 & 18.0 \\
\hline IE & 146.8 & 93.0 & 400.8 & 66.1 \\
\hline IL & 109.8 & 14.4 & 132.1 & 77.2 \\
\hline IN & 29.0 & 16.5 & 62.0 & 10.8 \\
\hline IT & 81.1 & 22.9 & 126.8 & 51.5 \\
\hline JP & 163.8 & 29.0 & 218.2 & 113.2 \\
\hline $\mathrm{KR}$ & 111.7 & 51.8 & 201.0 & 19.6 \\
\hline LU & 303.7 & 101.3 & 424.4 & 126.5 \\
\hline MX & 32.4 & 7.7 & 51.1 & 19.1 \\
\hline MY & 89.2 & 47.5 & 167.1 & 10.9 \\
\hline $\mathrm{NL}$ & 162.5 & 79.3 & 294.3 & 39.3 \\
\hline NO & 160.6 & 43.5 & 257.2 & 110.3 \\
\hline NZ & 102.5 & 59.7 & 201.3 & 26.1 \\
\hline PL & 54.2 & 22.3 & 86.3 & 20.4 \\
\hline PT & 127.5 & 48.8 & 231.6 & 59.3 \\
\hline $\mathrm{RU}$ & 55.9 & 29.4 & 103.9 & 14.9 \\
\hline SA & 42.1 & 11.3 & 68.6 & 25.5 \\
\hline SE & 148.1 & 48.1 & 256.0 & 99.4 \\
\hline SG & 116.3 & 27.0 & 178.1 & 66.1 \\
\hline TH & 89.0 & 38.5 & 181.9 & 26.9 \\
\hline TR & 39.2 & 24.7 & 95.5 & 14.8 \\
\hline US & 112.9 & 30.8 & 170.0 & 53.6 \\
\hline$Z A$ & 59.5 & 8.2 & 79.0 & 47.1 \\
\hline
\end{tabular}

China, Germany, France, Hong Kong, India, Netherlands, New Zealand, Turkey and the US. Concerning the order of integration of the series, the lowest estimate of $d$ is found in the case of Argentina, with a value of $\mathrm{d}$ of about 0.58 ; the correspondence confidence band does not include the value of 1 , which implies that the series is mean-reverting and shocks have transitory effects. By contrast, for all the other countries the confidence band includes 1 (even in a few cases, i.e. Austria and India, when the point estimate is below 1), and therefore shocks have permanent effects.

Table 4 focuses on the case of autocorrelated errors. The estimates of $\mathrm{d}$ are generally slightly smaller, but the same general conclusions are reached; in particular, Argentina is again the only
Table 3. Estimated values of $d$ with white noise errors.

\begin{tabular}{|c|c|c|c|}
\hline Country & No regressors & An intercept & $\begin{array}{c}\text { An intercept and a linear time } \\
\text { trend }\end{array}$ \\
\hline AR & $\begin{array}{c}0.73(0.61 \\
0.89)\end{array}$ & $\begin{array}{l}0.59(0.48 \\
0.79)\end{array}$ & $0.58(0.45,0.79)$ \\
\hline AT & $\begin{array}{c}0.95(0.85 \\
1.07)\end{array}$ & $\begin{array}{c}0.97(0.91 \\
1.05)\end{array}$ & $0.97(0.91,1.04)$ \\
\hline$A U$ & $\begin{array}{c}1.05(0.96 \\
1.17)\end{array}$ & $\begin{array}{c}1.35(1.27 \\
1.45)\end{array}$ & $1.35(1.27,1.45)$ \\
\hline $\mathrm{BE}$ & $\begin{array}{c}0.98(0.89 \\
1.10)\end{array}$ & $\begin{array}{c}1.09(1.01 \\
1.19)\end{array}$ & $1.09(1.01,1.20)$ \\
\hline$B R$ & $\begin{array}{c}0.94(0.79 \\
1.14)\end{array}$ & $\begin{array}{c}1.09(0.97 \\
1.26)\end{array}$ & $1.09(0.96,1.27)$ \\
\hline CA & $\begin{array}{c}1.03(0.95 \\
1.14)\end{array}$ & $\begin{array}{c}1.18(1.10 \\
1.28)\end{array}$ & $1.19(1.10,1.29)$ \\
\hline $\mathrm{CH}$ & $\begin{array}{c}1.01(0.93 \\
1.12)\end{array}$ & $\begin{array}{c}1.14(1.06 \\
1.23)\end{array}$ & $1.14(1.06,1.23)$ \\
\hline $\mathrm{CL}$ & $\begin{array}{c}1.04(0.94 \\
1.20)\end{array}$ & $\begin{array}{c}1.24(1.12 \\
1.40)\end{array}$ & $1.24(1.13,1.40)$ \\
\hline $\mathrm{CN}$ & $\begin{array}{c}1.05(0.97 \\
1.26)\end{array}$ & $\begin{array}{c}1.17(1.05 \\
1.35)\end{array}$ & $1.17(1.05,1.34)$ \\
\hline $\mathrm{CO}$ & $\begin{array}{c}1.05(0.91 \\
1.23)\end{array}$ & $\begin{array}{c}1.49(1.35 \\
1.68)\end{array}$ & $1.48(1.35,1.68)$ \\
\hline$C Z$ & $\begin{array}{c}0.96(0.83 \\
1.13)\end{array}$ & $\begin{array}{c}1.14(1.04 \\
1.30)\end{array}$ & $1.14(1.04,1.29)$ \\
\hline $\mathrm{DE}$ & $\begin{array}{c}1.03(0.95 \\
1.13)\end{array}$ & $\begin{array}{c}1.16(1.10 \\
1.24)\end{array}$ & $1.15(1.09,1.23)$ \\
\hline DK & $\begin{array}{c}1.00(0.91 \\
1.11)\end{array}$ & $\begin{array}{c}1.28(1.22 \\
1.36)\end{array}$ & $1.28(1.22,1.37)$ \\
\hline ES & $\begin{array}{c}1.11(1.03, \\
1.21)\end{array}$ & $\begin{array}{c}1.38(1.32 \\
1.44)\end{array}$ & $1.37(1.32,1.44)$ \\
\hline $\mathrm{FI}$ & $\begin{array}{c}1.01(0.91 \\
1.14)\end{array}$ & $\begin{array}{c}1.40(1.28 \\
1.56)\end{array}$ & $1.40(1.28,1.57)$ \\
\hline FR & $\begin{array}{c}1.01(0.92 \\
1.12)\end{array}$ & $\begin{array}{c}1.12(1.06 \\
1.21)\end{array}$ & $1.13(1.07,1.21)$ \\
\hline GB & $\begin{array}{c}1.02(0.94 \\
1.13)\end{array}$ & $\begin{array}{c}1.16(1.10 \\
1.23)\end{array}$ & $1.16(1.10,1.23)$ \\
\hline GR & $\begin{array}{c}1.11(1.03 \\
1.24)\end{array}$ & $\begin{array}{c}1.30(1.24 \\
1.37)\end{array}$ & $1.30(1.24,1.37)$ \\
\hline HK & $\begin{array}{c}1.10(1.00 \\
1.25)\end{array}$ & $\begin{array}{c}1.14(1.04 \\
1.29)\end{array}$ & $1.14(1.04,1.29)$ \\
\hline $\mathrm{HU}$ & $\begin{array}{c}1.08(1.00 \\
1.19)\end{array}$ & $\begin{array}{c}1.10(1.04 \\
1.19)\end{array}$ & $1.10(1.04,1.19)$ \\
\hline ID & $\begin{array}{c}1.12(0.98 \\
1.31)\end{array}$ & $\begin{array}{c}1.14(1.00 \\
1.33)\end{array}$ & $1.14(1.00,1.33)$ \\
\hline IE & $\begin{array}{c}1.00(0.91 \\
1.11)\end{array}$ & $\begin{array}{c}1.06(0.99 \\
1.16)\end{array}$ & $1.06(0.99,1.16)$ \\
\hline IL & $\begin{array}{c}0.97(0.86 \\
1.13)\end{array}$ & $\begin{array}{c}1.15(1.05 \\
1.30)\end{array}$ & $1.15(1.04,1.30)$ \\
\hline IN & $\begin{array}{c}0.96(0.90 \\
1.03)\end{array}$ & $\begin{array}{c}0.97(0.93 \\
1.03)\end{array}$ & $0.97(0.92,1.04)$ \\
\hline IT & $\begin{array}{c}1.03(0.96 \\
1.13)\end{array}$ & $\begin{array}{c}1.09(1.05 \\
1.15)\end{array}$ & $1.09(1.05,1.15)$ \\
\hline$J P$ & $\begin{array}{c}1.04(0.96 \\
1.14)\end{array}$ & $\begin{array}{c}1.30(1.23 \\
1.41)\end{array}$ & $1.30(1.22,1.41)$ \\
\hline KR & $\begin{array}{c}1.22(1.13 \\
1.33)\end{array}$ & $\begin{array}{c}1.30(1.21 \\
1.40)\end{array}$ & $1.29(1.20,1.40)$ \\
\hline LU & $\begin{array}{c}1.12(0.91 \\
1.41)\end{array}$ & $\begin{array}{c}1.71(1.43 \\
2.06)\end{array}$ & $1.70(1.42,2.06)$ \\
\hline MX & $\begin{array}{c}1.11(1.00 \\
1.25)\end{array}$ & $\begin{array}{c}1.24(1.15 \\
1.37)\end{array}$ & $1.24(1.15,1.37)$ \\
\hline MY & $\begin{array}{c}1.38(1.29 \\
1.50)\end{array}$ & $\begin{array}{c}1.43(1.33) \\
1.55)\end{array}$ & $1.43(1.33,1.55)$ \\
\hline NL & $\begin{array}{c}1.04(0.95 \\
1.16)\end{array}$ & $\begin{array}{c}1.23(1.15 \\
1.32)\end{array}$ & $1.23(1.15,1.31)$ \\
\hline NO & $\begin{array}{c}1.07(0.97 \\
1.19)\end{array}$ & $\begin{array}{c}1.34(1.25 \\
1.47)\end{array}$ & $1.34(1.25,1.46)$ \\
\hline NZ & $\begin{array}{c}1.07(0.96 \\
1.18)\end{array}$ & $\begin{array}{c}1.09(1.03 \\
1.17)\end{array}$ & $1.09(1.03,1.17)$ \\
\hline PL & $\begin{array}{c}0.97(0.80 \\
1.17)\end{array}$ & $\begin{array}{c}1.35(1.22 \\
1.53)\end{array}$ & $1.35(1.22,1.53)$ \\
\hline PT & $\begin{array}{c}1.10(1.02, \\
1.19)\end{array}$ & $\begin{array}{c}1.32(1.26 \\
1.39)\end{array}$ & $1.32(1.26,1.39)$ \\
\hline
\end{tabular}

(Continued) 
Table 3. (Continued).

\begin{tabular}{|c|c|c|c|}
\hline Country & No regressors & An intercept & $\begin{array}{l}\text { An intercept and a linear time } \\
\text { trend }\end{array}$ \\
\hline RU & $\begin{array}{c}1.04(0.88 \\
1.28)\end{array}$ & $\begin{array}{c}1.15(1.01, \\
1.35)\end{array}$ & $1.15(1.01,1.35)$ \\
\hline SA & $\begin{array}{c}1.16(1.00 \\
1.36)\end{array}$ & $\begin{array}{c}1.39(1.19, \\
1.64)\end{array}$ & $1.39(1.19,1.64)$ \\
\hline SE & $\begin{array}{c}1.02(0.94 \\
1.12)\end{array}$ & $\begin{array}{c}1.28(1.21, \\
1.38)\end{array}$ & $1.28(1.21,1.38)$ \\
\hline SG & $\begin{array}{c}1.08(0.99 \\
1.22)\end{array}$ & $\begin{array}{c}1.21(1.12, \\
1.34)\end{array}$ & $1.21(1.11,1.34)$ \\
\hline TH & $\begin{array}{c}1.24(1.17 \\
1.32)\end{array}$ & $\begin{array}{c}1.30(1.23, \\
1.41)\end{array}$ & $1.30(1.23,1.41)$ \\
\hline TR & $\begin{array}{c}1.04(0.94 \\
1.19)\end{array}$ & $\begin{array}{c}1.04(0.96 \\
1.16)\end{array}$ & $1.04(0.95,1.16)$ \\
\hline US & $\begin{array}{c}1.07(0.99 \\
1.17)\end{array}$ & $\begin{array}{c}1.32(1.26 \\
1.40)\end{array}$ & $1.31(1.25,1.37)$ \\
\hline ZA & $\begin{array}{c}1.01(0.91 \\
1.31)\end{array}$ & $\begin{array}{c}1.19(1.10, \\
1.31)\end{array}$ & $1.20(1.10,1.31)$ \\
\hline
\end{tabular}

The values in parentheses are the $95 \%$ confidence bands of the values of $d$. In bold, the selected specification on the basis of the statistical significance of the deterministic terms.

country for which evidence of mean reversion is found, the estimated value of $d$ being equal to 0.31 . The I(1) hypothesis cannot be rejected for 13 countries (South Africa, Indonesia, Russia, Luxembourg, Brazil, China, Hong Kong, Chile, Ireland, Turkey, Cech Republic, Israel and Finland); for the remaining 39 countries the estimates of $d$ are significantly higher than 1 . Table 5 shows a summary of the results.

In brief, mean reversion is only found in the case of Argentina, regardless of the assumption made about the behaviour of the errors; for four countries (Turkey, Ireland, Brazil and Indonesia) the unit root null hypothesis cannot be rejected in either case; finally, for another 27 countries $\mathrm{d}$ is statistically higher than 1 in both cases considered. On the whole, the results are robust to the specification adopted for the error term.

To make the findings for different countries more directly comparable, we also re-estimate the model over the longest span of data available for all countries, namely starting in 1999Q1 (the start date for the Luxembourg series, the shortest one); these results are displayed in Table A1 and A2 in the Appendix for the two cases of white noise and autocorrelated errors, respectively. As before, all the estimated values of $\mathrm{d}$ are equal to or higher than 1 except for Argentina, for which mean reversion is found with autocorrelated errors.
Table 4. Estimated values of $d$ with autocorrelated errors.

\begin{tabular}{|c|c|c|c|}
\hline Country & No regressors & An intercept & $\begin{array}{c}\text { An intercept and a linear time } \\
\text { trend }\end{array}$ \\
\hline AR & $\begin{array}{c}0.59(0.44 \\
0.77)\end{array}$ & $\begin{array}{c}0.38(0.27 \\
0.54)\end{array}$ & $0.31(0.17,0.50)$ \\
\hline AT & $\begin{array}{c}0.89(0.70 \\
1.14)\end{array}$ & $\begin{array}{c}1.17(1.06 \\
1.34)\end{array}$ & $1.17(1.05,1.31)$ \\
\hline$A U$ & $\begin{array}{c}0.98(0.82 \\
1.16)\end{array}$ & $\begin{array}{c}1.45(1.29 \\
1.68)\end{array}$ & $1.45(1.30,1.66)$ \\
\hline BE & $\begin{array}{c}0.93(0.80 \\
1.16)\end{array}$ & $\begin{array}{c}1.17(1.02 \\
1.44)\end{array}$ & $1.19(1.02,1.44)$ \\
\hline$B R$ & $\begin{array}{c}0.79(0.55 \\
1.13)\end{array}$ & $\begin{array}{c}1.06(0.86 \\
1.40)\end{array}$ & $1.05(0.83,1.41)$ \\
\hline CA & $\begin{array}{c}0.98(0.85 \\
1.16)\end{array}$ & $\begin{array}{c}1.21(1.06 \\
1.47)\end{array}$ & $1.24(1.08,1.47)$ \\
\hline $\mathrm{CH}$ & $\begin{array}{c}0.98(0.84 \\
1.17)\end{array}$ & $\begin{array}{c}1.23(1.09 \\
1.44)\end{array}$ & $1.24(1.08,1.44)$ \\
\hline $\mathrm{CL}$ & $\begin{array}{c}0.99(0.84 \\
1.26)\end{array}$ & $\begin{array}{c}1.07(0.91 \\
1.32)\end{array}$ & $1.07(0.90,1.32)$ \\
\hline $\mathrm{CN}$ & $\begin{array}{c}0.94(0.76 \\
1.20)\end{array}$ & $\begin{array}{c}1.04(0.90 \\
1.33)\end{array}$ & $1.06(0.87,1.34)$ \\
\hline $\mathrm{CO}$ & $\begin{array}{c}0.97(0.72 \\
1.30)\end{array}$ & $\begin{array}{c}1.59(1.29 \\
2.16)\end{array}$ & $1.65(1.30,2.04)$ \\
\hline$C Z$ & $\begin{array}{c}0.86(0.66 \\
1.14)\end{array}$ & $\begin{array}{c}1.09(0.93 \\
1.30)\end{array}$ & $1.09(0.93,1.29)$ \\
\hline $\mathrm{DE}$ & $\begin{array}{c}1.04(0.91 \\
1.22)\end{array}$ & $\begin{array}{c}1.40(1.27 \\
1.61)\end{array}$ & $1.37(1.25,1.58)$ \\
\hline DK & $\begin{array}{c}0.98(0.83 \\
1.18)\end{array}$ & $\begin{array}{c}1.50(1.35 \\
1.70)\end{array}$ & $1.51(1.36,1.72)$ \\
\hline ES & $\begin{array}{c}1.15(1.02 \\
1.34)\end{array}$ & $\begin{array}{c}1.74(1.62, \\
1.92)\end{array}$ & $1.75(1.62,1.91)$ \\
\hline $\mathrm{FI}$ & $\begin{array}{c}0.89(0.74 \\
1.12)\end{array}$ & $\begin{array}{c}1.15(0.99 \\
1.35)\end{array}$ & $1.15(0.99,1.35)$ \\
\hline FR & $\begin{array}{c}1.00(0.87 \\
1.18)\end{array}$ & $\begin{array}{c}1.28(1.16 \\
1.49)\end{array}$ & $1.30(1.18,1.48)$ \\
\hline GB & $\begin{array}{c}1.02(0.89 \\
1.21)\end{array}$ & $\begin{array}{c}1.32(1.22 \\
1.46)\end{array}$ & $1.32(1.22,1.48)$ \\
\hline GR & $\begin{array}{c}1.15(1.02, \\
1.35)\end{array}$ & $\begin{array}{c}1.56(1.44 \\
1.75)\end{array}$ & $1.55(1.43,1.73)$ \\
\hline HK & $\begin{array}{c}1.01(0.86 \\
1.21)\end{array}$ & $\begin{array}{c}1.05(0.91 \\
1.32)\end{array}$ & $1.06(0.90,1.31)$ \\
\hline HU & $\begin{array}{c}1.09(0.97 \\
1.27)\end{array}$ & $\begin{array}{c}1.21(1.10 \\
1.35)\end{array}$ & $1.21(1.10,1.35)$ \\
\hline ID & $\begin{array}{c}0.78(0.63 \\
1.02)\end{array}$ & $\begin{array}{c}0.77(0.60 \\
1.02)\end{array}$ & $0.76(0.62,1.02)$ \\
\hline IE & $\begin{array}{c}0.95(0.84 \\
1.11)\end{array}$ & $\begin{array}{c}1.07(0.95 \\
1.23)\end{array}$ & $1.07(0.95,1.23)$ \\
\hline IL & $\begin{array}{c}0.90(0.72 \\
1.16)\end{array}$ & $\begin{array}{c}1.11(0.97 \\
1.32)\end{array}$ & $1.10(0.97,1.30)$ \\
\hline IN & $\begin{array}{c}1.06(0.96 \\
1.21)\end{array}$ & $\begin{array}{c}1.20(1.11 \\
1.32)\end{array}$ & $1.20(1.11,1.32)$ \\
\hline IT & $\begin{array}{c}1.10(0.96 \\
1.26)\end{array}$ & $\begin{array}{c}1.49(1.38 \\
1.64)\end{array}$ & $1.48(1.38,1.63)$ \\
\hline$J P$ & $\begin{array}{c}1.03(0.91 \\
1.25)\end{array}$ & $\begin{array}{c}1.20(1.09 \\
1.32)\end{array}$ & $1.18(1.09,1.31)$ \\
\hline KR & $\begin{array}{c}1.24(1.03 \\
1.50)\end{array}$ & $\begin{array}{c}1.41(1.19 \\
1.68)\end{array}$ & $1.40(1.18,1.68)$ \\
\hline LU & $\begin{array}{c}0.51(0.40 \\
1.10)\end{array}$ & $\begin{array}{c}1.04(0.79 \\
1.66)\end{array}$ & $1.04(0.72,1.66)$ \\
\hline MX & $\begin{array}{c}1.04(0.86 \\
1.31)\end{array}$ & $\begin{array}{c}1.43(1.19 \\
1.80)\end{array}$ & $1.43(1.19,1.81)$ \\
\hline MY & $\begin{array}{c}1.35(1.16 \\
1.62)\end{array}$ & $\begin{array}{c}1.37(1.17 \\
1.62)\end{array}$ & $1.37(1.17,1.62)$ \\
\hline NL & $\begin{array}{c}0.97(0.79 \\
1.18)\end{array}$ & $\begin{array}{c}1.32(1.19 \\
1.50)\end{array}$ & $1.33(1.19,1.49)$ \\
\hline NO & $\begin{array}{c}0.98(0.82 \\
1.19)\end{array}$ & $\begin{array}{c}1.31(1.08 \\
1.64)\end{array}$ & $1.31(1.09,1.61)$ \\
\hline NZ & $\begin{array}{c}1.10(0.95 \\
1.28)\end{array}$ & $\begin{array}{c}1.31(1.17 \\
1.48)\end{array}$ & $1.32(1.18,1.49)$ \\
\hline PL & $\begin{array}{c}0.67(0.54 \\
1.02)\end{array}$ & $\begin{array}{c}1.18(1.01 \\
1.43)\end{array}$ & $1.19(1.01,1.43)$ \\
\hline PT & $\begin{array}{c}1.13(1.00 \\
1.28)\end{array}$ & $\begin{array}{c}1.50(1.36 \\
1.66)\end{array}$ & $1.50(1.37,1.67)$ \\
\hline
\end{tabular}

(Continued) 
Table 4. (Continued).

\begin{tabular}{|c|c|c|c|}
\hline Country & No regressors & An intercept & $\begin{array}{l}\text { An intercept and a linear time } \\
\text { trend }\end{array}$ \\
\hline RU & $\begin{array}{c}0.73(0.61 \\
1.01)\end{array}$ & $\begin{array}{c}0.91(0.78 \\
1.20)\end{array}$ & $0.88(0.58,1.22)$ \\
\hline SA & $\begin{array}{c}0.94(0.61 \\
1.39)\end{array}$ & $\begin{array}{c}0.84(0.60 \\
1.34)\end{array}$ & $0.83(0.47,1.35)$ \\
\hline SE & $\begin{array}{c}1.02(0.91 \\
1.20)\end{array}$ & $\begin{array}{c}1.38(1.22, \\
1.63)\end{array}$ & $1.39(1.21,1.63)$ \\
\hline SG & $\begin{array}{c}1.09(0.90 \\
1.33)\end{array}$ & $\begin{array}{c}1.20(1.01, \\
1.78)\end{array}$ & $1.19(1.01,1.46)$ \\
\hline TH & $\begin{array}{c}1.35(1.20 \\
1.55)\end{array}$ & $\begin{array}{c}1.51(1.30, \\
1.72)\end{array}$ & $1.50(1.29,1.71)$ \\
\hline TR & $\begin{array}{c}0.97(0.85 \\
1.17)\end{array}$ & $\begin{array}{c}1.08(0.96 \\
1.27)\end{array}$ & $1.08(0.95,1.29)$ \\
\hline US & $\begin{array}{c}1.03(0.89 \\
1.22)\end{array}$ & $\begin{array}{c}1.82(1.64, \\
2.12)\end{array}$ & $1.74(1.58,2.00)$ \\
\hline ZA & $\begin{array}{c}0.90(0.77 \\
1.11)\end{array}$ & $\begin{array}{c}1.21(1.01, \\
1.47)\end{array}$ & $1.21(1.01,1.47)$ \\
\hline
\end{tabular}

The values in parentheses are the $95 \%$ confidence bands of the values of $d$. In bold, the selected specification on the basis of the statistical significance of the deterministic terms.

\section{Conclusions}

This paper investigates the degree of persistence of the private debt-to-GDP ratio in 43 OECD countries by estimating the fractional integration parameter of each series. Almost all of them are found to be highly persistent, with orders of integration around or above 1 . The only exception is Argentina, where the series appears to be meanreverting. This reflects the relatively unique experience of this country, who underwent an economic depression from 1998 to 2002, which was followed by a significant episode of deleveraging between 2002 and 2008 (one of the 45 main such episodes since 1930 identified by the McKinsey Global Institute).

On the whole, the results highlight the key importance of macroprudential policy as one of the pillars of macro policy. They suggest longlived effects of shocks to the private debt-to-GDP ratio which require appropriate policy actions. In the specific case of the GFC credit rationing was typically accompanied by increased collateralization with significant and adverse lasting effects on the deleveraging process and economic growth.

Table 5. Summary table.

\begin{tabular}{|c|c|c|c|c|c|}
\hline \multicolumn{3}{|c|}{ No autocorrelation } & \multicolumn{3}{|c|}{ Autocorrelation } \\
\hline $\begin{array}{l}\text { Mean reversion } \\
d<1 \\
A R(0.59)\end{array}$ & $\begin{array}{l}\text { Unit roots } \\
\quad \mathrm{d}=1 \\
\text { AT }(0.97) \\
\text { IN }(0.97) \\
\text { TR }(1.04) \\
\text { IE (1.06) } \\
\text { BR (1.09) } \\
\text { ID (1.14) }\end{array}$ & $\begin{array}{c}\text { Explosive } \\
\text { d }>1 \\
\text { BE }(1.09) \\
\text { IT }(1.09) \\
\text { NZ (1.09) } \\
\text { HU (1.10) } \\
\text { FR (1.13) } \\
\text { CH (1.14) } \\
\text { CZ (1.14) } \\
\text { HK (1.14) } \\
\text { DE (1.15) } \\
\text { RU (1.15) } \\
\text { IL (1.15) } \\
\text { GB }(1.16) \\
\text { CN (1.17) } \\
\text { CA (1.19) } \\
\text { ZA (1.19) } \\
\text { SG (1.21) } \\
\text { NL (1.23) } \\
\text { CL (1.24) } \\
\text { MX (1.24) } \\
\text { DK (1.28) } \\
\text { SE (1.28) } \\
\text { GR (1.30) } \\
\text { JP (1.30) } \\
\text { KR (1.30) } \\
\text { TH (1.30) } \\
\text { US (1.31) } \\
\text { PT (1.32) } \\
\text { NO (1.34) } \\
\text { AU (1.35) } \\
\text { PL (1.35) } \\
\text { ES (1.38) } \\
\text { SA (1.39) } \\
\text { FI (1.40) } \\
\text { MY (1.43) } \\
\text { CO (1.49) } \\
\text { LU (1.71) }\end{array}$ & $\begin{array}{c}\text { Mean reversion } \\
\mathrm{d}<1 \\
\text { AR (0.31) }\end{array}$ & $\begin{array}{l}\text { Unit roots } \\
\text { d = 1 } \\
\text { ID }(0.77) \\
\text { SA (0.83) } \\
\text { RU (0.88) } \\
\text { LU (1.04) } \\
\text { BR (1.06) } \\
\text { CN (1.06) } \\
\text { HK (1.06) } \\
\text { CL (1.07) } \\
\text { IE (1.07) } \\
\text { TR (1.08) } \\
\text { CZ (1.09) } \\
\text { IL (1.11) } \\
\text { FI (1.15) }\end{array}$ & $\begin{array}{c}\text { Explosive } \\
\text { d }>1 \\
\text { AT }(1.17) \\
\text { BE (1.17) } \\
\text { PL (1.18) } \\
\text { IN (1.20) } \\
\text { SG (1.20) } \\
\text { JP (1.20) } \\
\text { HU (1.21) } \\
\text { ZA (1.21) } \\
\text { CH }(1.23) \\
\text { CA (1.24) } \\
\text { FR (1.30) } \\
\text { NO (1.31) } \\
\text { NZ (1.31) } \\
\text { GB (1.32) } \\
\text { NL (1.32) } \\
\text { MY (1.37) } \\
\text { SE (1.38) } \\
\text { MX (1.43) } \\
\text { AU (1.45) } \\
\text { IT (1.49) } \\
\text { PT (1.50) } \\
\text { TH (1.51) } \\
\text { GR (1.56) } \\
\text { DE (1.40) } \\
\text { KR (1.41) } \\
\text { DK (1.50) } \\
\text { CO (1.59) } \\
\text { ES (1.74) } \\
\text { US (1.82) }\end{array}$ \\
\hline
\end{tabular}


This called for a clear focus of central banks on the stability of the financial system. Prompt measures aimed at attenuating the deleveraging process and reducing the severity of the credit crunch would have been extremely beneficial and mitigated the effects of the GFC on the real sector. This is an important policy lesson to be learned to tackle future crises more effectively.

The analysis carried out in this paper can be extended in several ways. First, the presence of structural breaks, such as the GFC can be examined using various methods including endogenous/exogenous break tests, rolling and/or recursive methods, sub-sample estimation. Nonlinearities can also be analysed using approaches as the one proposed in Cuestas and Gil-Alana (2016) and based on Chebyshev polynomials in time. Further work can be done distinguishing between private debt held by households and by non-financial corporations (NFCs) respectively; this is particularly interesting given the crucial role played by household leverage in bringing about the GFC - according to Mian and Sufi (2010), while the total debt to income ratio increased by $0.8 \%$, total mortgage debt grew by $34 \%$ from 2002 to 2006.

A final interesting issue is the link between private and public debt and economic activity. This has been examined Batini, Melina, and Villa (2019), who build on the work of Mian and Sufi (2010), in the context of a theoretical model in which the government provides financial assistance to credit-constrained agents to reduce deleveraging; using calibration and simulation techniques they show that higher private debt makes recessions more severe, whilst increases in public debt only worsen them when its level is particularly high by reducing fiscal accommodation during periods of private deleveraging. Future research could revisit these issues empirically by estimating an appropriate econometric model.

\section{Acknowledgments}

Prof. Luis A. Gil-Alana gratefully acknowledges financial support from the MINEIC-AEI-FEDER ECO2017-85503-R project from 'Ministerio de Economía, Industria y Competitividad' (MINEIC), 'Agencia Estatal de
Investigación' (AEI) Spain and 'Fondo Europeo de Desarrollo Regional' (FEDER).

Comments from the Editor and an anonymous reviewer are gratefully acknowledged.

\section{Disclosure statement}

No potential conflict of interest was reported by the author(s).

\section{Funding}

This work was supported by the Ministerio de Economía, Industria y Competitividad, Gobierno de España [MINEICAEI-FEDER ECO2017-85503-R].

\section{ORCID}

Guglielmo Maria Caporale (D) http://orcid.org/0000-00020144-4135

Luis Alberiko Gil-Alana (D) http://orcid.org/0000-0002-57603123

\section{References}

Andrés, J., O. Arce, D. Thaler, and C. Thomas. 2020. "When Fiscal Consolidation Meets Private Deleveraging." Review of Economic Dynamics 37: 214-233. doi:10.1016/j. red.2020.02.002.

Batini, N., G. Melina, and S. Villa. 2019. "Fiscal Buffers, Private Debt, and Recession: The Good, the Bad and the Ugly." Journal of Macroeconomics 62 (C): 103044. doi:10.1016/j.jmacro.2018.06.012.

Bole, V., J. Prašnikar, and D. Trobec. 2014. "Policy Measures in the Deleveraging Process: A Macroprudential Evaluation." Journal of Policy Modeling 36 (2): 410-432. doi:10.1016/j.jpolmod.2014.01.007.

Brzoza-Brzezina, M., M. Kolasa, and K. Makarski. 2015. "Macroprudential Policy and Imbalances in the Euro Area." Journal of International Money and Finance 51: 137-154. doi:10.1016/j.jimonfin.2014.10.004.

Chen, S., M. Kim, M. Otte, K. Wiseman, and A. Zdzienicka. 2015. "Private Sector Deleveraging and Growth following Busts." doi: 10.2139/ssrn.2572489.

Cuestas, J. C., and L. A. Gil-Alana. 2016. "Testing for Long-Memory in the Presence of Non-Linear Chebyshev Polynomials." Studies in Nonlinear Dynamics and Econometrics 20 (1): 57-74.

Dahlhaus, R. 1989. "Efficient Parameter Estimation for Self-similar Processes." Annals of Statistics 17 (4): 1749-1766. doi:10.1214/aos/1176347393.

De Blas, B., and M. Malmierca. 2020. "Financial Frictions and Stabilization Policies.” Economic Modelling 89: 166-188. doi:10.1016/j.econmod.2019.10.019. 
Dehmej, S., and L. Gambacorta (2017): "Macroprudential Policy in a Monetary Union.” Working Paper No. 3, Bank Al-Maghrib.

Dickey, D. A., and W. A. Fuller. 1979. "Distributions of the Estimators for Autoregressive Time Series with a Unit Root." Journal of American Statistical Association 74 (366): 427-481.

Eggertsson, G. T., and P. Krugman. 2012. "Debt, Deleveraging and the Liquidity Trap. A Fisher-Minsky-Koo Approach." The Quarterly Journal of Economics 127 (3): 1469-1513. doi:10.1093/qje/qjs023.

Elliot, G., . T. J., T. J. Rothenberg, and J. H. Stock. 1996. "Efficient tests for an autoregressive unit root." Econometrica 64 (4): 813-836. doi:10.2307/2171846.

Estrada, A., E. Valdeolivas, J. Vallés, and D. Garrote. 2014, "Household debt and uncertainty: Private consumption after the great recession." Working Paper, 1415, Bank of Spain

Gil-Alana, L. A. 2000. “Evaluation of Robinson's (1997) Tests in Finite Sample." Journal of Statistical Computation and Simulation 68 (1): 39-64. doi:10.1080/00949650008812055.

Gil-Alana, L. A., and P. M. Robinson. 1997. "Testing of Unit Roots and Other Nonstationary Hypotheses in Macroeconomic Time Series." Journal of Econometrics 80 (2): 241-268. doi:10.1016/S0304-4076(97)00038-9.

Ivens, A. 2018. "Optimal Fiscal Policy under Private Debt Deleveraging." Journal of Economic Dynamics \& Control 97: 1-18. doi:10.1016/j.jedc.2018.09.003.

Kiyotaki, N., and J. Moore. 2002. "Balance Sheet Contagion." American Economic Review 92 (2): 46-50. doi:10.1257/ 000282802320188989.

Koo, R. 2008. The Holy Grail of Macroeconomics: Lessons from Japan's Great Recession. Wiley. USA: Hoboken.

Kwiatkowski, D., P. C. D. Phillips, P. Schmidt, and Y. Shin. 1992. "Testing the Null Hypothesis of Stationarity against the Alternative of a Unit Root: How Sure are We that Economic Time Series Have a Unit Root?" Journal of
Econometrics 54 (1-3): 159-178. doi:10.1016/03044076(92)90104-Y.

Mian, A., and A. Sufi. 2010. "House Prices, Home Equity-Based Borrowing, and the U.S. Household Leverage Crisis." American Economic Review 5 (101): 2132-2156.

Ng, S., and P. Perron. 2001. "Lag Length Selection and the Construction of Unit Root Tests with Good Size and Power.” Econometrica 69 (6): 519-1554. doi:10.1111/14680262.00256.

Phillips, P. C. B., and P. Perron. 1988. “Testing for a Unit Root in Time Series Regression.” Biometrika 75 (2): 335-346. doi:10.1093/biomet/75.2.335.

Quint, D., and Q. Rabanal. 2014. "Monetary and Macroprudential Policy in an Estimated DSGE Model of the Euro Area." International Journal of Central Banking 10 (2): 169-236.

Raberto, M., A. Teglio, and S. Cincotti. 2012. "Debt, Deleveraging and Business Cycles: An Agent-based Perspective, Economics: The Open-Access, Open-Assessment E-Journal, 1864-6042." Kiel Institute for the World Economy (Ifw), Kiel 6 (2012-27): 1-49. doi:10.5018/economics-ejournal.ja.2012-27..

Ramalho, J. J., and J. V. Silva. 2009. "A Two-part Fractional Regression Model for the Financial Leverage Decisions of Micro, Small, Medium and Large Firms." Quantitative Finance 9 (5): 621-636. doi:10.1080/ 14697680802448777.

Robinson, P. M. 1994. "Efficient Tests of Nonstationary Hypotheses." Journal of the American Statistical Association 89 (428): 1420-1437. doi:10.1080/ 01621459.1994 .10476881$.

Rubio, M., and J. A. Carrasco-Gallego. 2014. "Macroprudential and Monetary Policies: Implications for Financial Stability and Welfare." Journal of Banking and Finance 49: 326-336. doi:10.1016/j. jbankfin.2014.02.012. 


\section{APPENDIX}

Table A1: Estimated values of d with white noise errors, 1999Q1-2020Q1.

\begin{tabular}{|c|c|c|c|}
\hline Country & No regressors & An intercept & An intercept and a linear time trend \\
\hline AR & $1.02(0.86,1.28)$ & $1.16(0.89,1.60)$ & $1.17(0.89,1.60)$ \\
\hline AT & $0.99(0.85,1.07)$ & $1.00(0.86,1.19)$ & $1.00(0.89,1.16)$ \\
\hline AU & $0.97(0.83,1.16)$ & $1.42(1.30,1.59)$ & $1.40(1.28,1.57)$ \\
\hline $\mathrm{BE}$ & $0.97(0.82,1.16)$ & $1.06(0.90,1.26)$ & $1.05(0.90,1.25)$ \\
\hline$B R$ & $0.93(0.78,1.12)$ & $1.05(0.93,1.22)$ & $1.05(0.93,1.22)$ \\
\hline CA & $0.95(0.80,1.15)$ & $1.16(1.03,1.36)$ & $1.17(1.03,1.36)$ \\
\hline $\mathrm{CH}$ & $0.97(0.83,1.18)$ & $1.10(0.99,1.27)$ & $1.11(0.99,1.29)$ \\
\hline $\mathrm{CL}$ & $1.08(0.94,1.28)$ & $1.27(1.12,1.48)$ & $1.28(1.13,1.48)$ \\
\hline $\mathrm{CN}$ & $1.05(0.90,1.28)$ & $1.21(1.03,1.49)$ & $1.21(1.04,1.46)$ \\
\hline $\mathrm{CO}$ & $0.97(0.82,1.20)$ & $1.44(1.31,1.63)$ & $1.43(1.31,1.63)$ \\
\hline $\mathrm{CZ}$ & $0.91(0.76,1.13)$ & $1.17(1.07,1.33)$ & $1.17(1.07,1.32)$ \\
\hline $\mathrm{DE}$ & $1.00(0.88,1.19)$ & $1.43(1.30,1.63)$ & $1.42(1.29,1.60)$ \\
\hline DK & $0.95(0.80,1.14)$ & $1.30(1.21,1.43)$ & $1.29(1.20,1.42)$ \\
\hline ES & $1.09(0.97,1.26)$ & $1.48(1.40,1.59)$ & $1.43(1.37,1.53)$ \\
\hline $\mathrm{FI}$ & $0.97(0.81,1.20)$ & $1.16(0.97,1.48)$ & $1.16(0.97,1.47)$ \\
\hline FR & $0.98(0.84,1.18)$ & $1.19(1.02,1.47)$ & $1.20(1.03,1.42)$ \\
\hline GB & $0.99(0.87,1.18)$ & $1.22(1.12,1.35)$ & $1.20(1.12,1.32)$ \\
\hline GR & $1.03(0.91,1.20)$ & $1.37(1.28,1.48)$ & $1.34(1.26,1.45)$ \\
\hline HK & $0.96(0.80,1.19)$ & $1.08(0.97,1.27)$ & $1.09(0.96,1.28)$ \\
\hline $\mathrm{HU}$ & $1.05(0.92,1.22)$ & $1.09(1.00,1.21)$ & $1.08(1.00,1.21)$ \\
\hline ID & $0.65(0.52,0.83)$ & $1.00(0.86,1.17)$ & $1.00(0.87,1.17)$ \\
\hline IE & $0.97(0.84,1.17)$ & $1.04(0.93,1.20)$ & $1.04(0.93,1.20)$ \\
\hline IL & $0.97(0.85,1.16)$ & $1.20(1.05,1.43)$ & $1.20(1.05,1.42)$ \\
\hline IN & $0.93(0.79,1.10)$ & $0.94(0.86,1.04)$ & $0.94(0.87,1.04)$ \\
\hline IT & $1.01(0.87,1.21)$ & $1.29(1.21,1.41)$ & $1.27(1.19,1.36)$ \\
\hline$J P$ & $0.97(0.83,1.16)$ & $1.27(1.14,1.48)$ & $1.26(1.13,1.46)$ \\
\hline $\mathrm{KR}$ & $0.92(0.76,1.12)$ & $1.43(1.29,1.61)$ & $1.42(1.29,1.59)$ \\
\hline LU & $1.12(0.91,1.41)$ & $1.72(1.43,2.06)$ & $1.71(1.42,2.05)$ \\
\hline$M X$ & $0.96(0.79,1.23)$ & $1.07(0.97,1.22)$ & $1.07(0.97,1.23)$ \\
\hline MY & $0.93(0.80,1.13)$ & $1.40(1.23,1.63)$ & $1.37(1.22,1.60)$ \\
\hline $\mathrm{NL}$ & $0.95(0.80,1.15)$ & $1.27(1.16,1.43)$ & $1.27(1.16,1.42)$ \\
\hline NO & $0.98(0.81,1.20)$ & $1.30(1.14,1.53)$ & $1.30(1.14,1.52)$ \\
\hline NZ & $0.97(0.84,1.16)$ & $1.38(1.27,1.55)$ & $1.37(1.26,1.54)$ \\
\hline $\mathrm{PL}$ & $1.00(0.84,1.23)$ & $1.30(1.15,1.54)$ & $1.29(1.14,1.52)$ \\
\hline PT & $1.03(0.93,1.19)$ & $1.57(1.46,1.76)$ & $1.51(1.41,1.66)$ \\
\hline $\mathrm{RU}$ & $0.93(0.75,1.17)$ & $1.14(1.02,1.33)$ & $1.15(1.02,1.33)$ \\
\hline SA & $1.01(0.84,1.24)$ & $1.39(1.19,1.65)$ & $1.39(1.19,1.66)$ \\
\hline SE & $0.95(0.80,1.14)$ & $1.17(1.05,1.33)$ & $1.17(1.05,1.32)$ \\
\hline SG & $0.98(0.84,1.18)$ & $1.24(1.09,1.44)$ & $1.25(1.11,1.45)$ \\
\hline $\mathrm{TH}$ & $0.90(0.75,1.10)$ & $1.36(1.26,1.49)$ & $1.32(1.23,1.43)$ \\
\hline TR & $0.93(0.77,1.17)$ & $1.02(0.92,1.17)$ & $1.02(0.90,1.19)$ \\
\hline US & $1.00(0.86,1.19)$ & $1.53(1.41,1.70)$ & $1.48(1.38,1.62)$ \\
\hline ZA & $0.96(0.82,1.18)$ & $1.14(1.02,1.29)$ & $1.14(1.02,1.29)$ \\
\hline
\end{tabular}

The values in parentheses are the $95 \%$ confidence bands of the values of $d$. In bold, the selected specification on the basis of the statistical significance of the deterministic terms. 
Table A2: Estimated values of d with autocorrelated errors, 1999Q1-2020Q1.

\begin{tabular}{|c|c|c|c|}
\hline Country & No regressors & An intercept & An intercept and a linear time trend \\
\hline AR & $0.73(0.53,1.01)$ & $0.47(0.33,0.69)$ & $0.39(0.17,0.71)$ \\
\hline AT & $0.92(0.69,1.28)$ & $1.00(0.66,1.32)$ & $1.02(0.81,1.26)$ \\
\hline AU & $0.89(0.64,1.23)$ & $1.58(1.27,2.10)$ & $1.51(1.25,2.13)$ \\
\hline $\mathrm{BE}$ & $0.87(0.50,1.24)$ & $1.08(0.75,1.64)$ & $1.05(0.70,1.58)$ \\
\hline BR & $0.85(0.58,1.28)$ & $1.11(0.89,1.51)$ & $1.11(0.86,1.51)$ \\
\hline CA & $0.82(0.55,1.16)$ & $1.09(0.90,1.58)$ & $1.11(0.82,1.58)$ \\
\hline $\mathrm{CH}$ & $0.86(0.62,1.20)$ & $1.11(0.96,1.41)$ & $1.14(0.94,1.50)$ \\
\hline $\mathrm{CL}$ & $0.98(0.74,1.33)$ & $1.23(0.98,1.65)$ & $1.24(0.96,1.63)$ \\
\hline $\mathrm{CN}$ & $0.85(0.59,1.25)$ & $1.01(0.84,1.52)$ & $1.01(0.78,1.47)$ \\
\hline $\mathrm{CO}$ & $0.77(0.49,1.15)$ & $1.48(1.28,1.94)$ & $1.49(1.28,1.96)$ \\
\hline$C Z$ & $0.75(0.46,1.14)$ & $1.19(1.01,1.43)$ & $1.20(1.01,1.41)$ \\
\hline $\mathrm{DE}$ & $0.96(0.73,1.27)$ & $1.45(1.20,1.86)$ & $1.45(1.20,1.83)$ \\
\hline DK & $0.87(0.59,1.19)$ & $1.46(1.28,1.72)$ & $1.43(1.26,1.69)$ \\
\hline ES & $1.06(0.86,1.31)$ & $1.77(1.61,2.06)$ & $1.67(1.54,1.89)$ \\
\hline $\mathrm{FI}$ & $0.73(0.23,1.14)$ & $0.86(0.75,1.15)$ & $0.81(0.56,1.15)$ \\
\hline$F R$ & $0.88(0.60,1.22)$ & $1.11(0.94,1.52)$ & $1.11(0.86,1.53)$ \\
\hline GB & $0.95(0.73,1.24)$ & $1.38(1.19,1.64)$ & $1.34(1.18,1.52)$ \\
\hline GR & $1.01(0.77,1.33)$ & $1.64(1.46,2.09)$ & $1.59(1.41,2.10)$ \\
\hline HK & $0.76(0.52,1.18)$ & $0.94(0.82,1.13)$ & $0.91(0.72,1.16)$ \\
\hline $\mathrm{HU}$ & $1.02(0.82,1.28)$ & $1.20(1.06,1.39)$ & $1.20(1.06,1.37)$ \\
\hline ID & $0.71(0.45,1.16)$ & $1.29(1.09,1.60)$ & $1.27(1.08,1.93)$ \\
\hline IE & $0.86(0.51,1.10)$ & $1.02(0.84,1.24)$ & $1.02(0.83,1.25)$ \\
\hline IL & $0.95(0.61,1.27)$ & $1.02(0.82,1.30)$ & $1.02(0.83,1.28)$ \\
\hline IN & $0.93(0.65,1.30)$ & $1.35(1.17,1.60)$ & $1.30(1.14,1.66)$ \\
\hline IT & $0.92(0.65,1.27)$ & $1.54(1.40,1.77)$ & $1.48(1.35,1.64)$ \\
\hline$J P$ & $0.90(0.66,1.25)$ & $1.02(0.79,1.29)$ & $1.00(0.82,1.26)$ \\
\hline $\mathrm{KR}$ & $0.81(0.50,1.17)$ & $1.58(1.14,2.41)$ & $1.47(1.15,2.53)$ \\
\hline LU & $0.51(0.40,1.13)$ & $1.04(0.79,1.65)$ & $1.03(0.72,1.64)$ \\
\hline MX & $0.63(0.39,1.17)$ & $1.02(0.89,1.19)$ & $1.01(0.83,1.20)$ \\
\hline MY & $0.83(0.56,1.14)$ & $1.17(0.95,1.58)$ & $1.16(0.97,1.46)$ \\
\hline $\mathrm{NL}$ & $0.81(0.47,1.19)$ & $1.34(1.13,1.63)$ & $1.34(1.13,1.62)$ \\
\hline NO & $0.77(0.28,1.20)$ & $1.15(0.84,1.88)$ & $1.11(0.79,1.75)$ \\
\hline NZ & $0.93(0.68,1.25)$ & $1.41(1.19,1.74)$ & $1.39(1.18,1.75)$ \\
\hline $\mathrm{PL}$ & $0.74(0.40,1.18)$ & $1.09(0.86,1.41)$ & $1.08(0.83,1.40)$ \\
\hline PT & $0.96(0.73,1.27)$ & $1.56(1.39,1.84)$ & $1.48(1.35,1.69)$ \\
\hline RU & $0.63(0.52,1.05)$ & $1.01(0.82,1.37)$ & $1.01(0.62,1.37)$ \\
\hline SA & $0.79(0.40,1.29)$ & $0.92(0.64,1.42)$ & $0.94(0.54,1.42)$ \\
\hline SE & $1.86(0.55,1.23)$ & $1.41(1.09,1.86)$ & $1.41(1.08,1.86)$ \\
\hline SG & $0.90(0.66,1.29)$ & $1.17(0.92,1.62)$ & $1.16(0.93,1.62)$ \\
\hline $\mathrm{TH}$ & $0.71(1.47,1.08)$ & $1.55(1.32,1.85)$ & $1.47(1.30,1.88)$ \\
\hline TR & $0.73(0.61,1.04)$ & $1.04(0.90,1.29)$ & $1.03(0.84,1.30)$ \\
\hline US & $0.93(0.70,1.26)$ & $1.87(1.58,2.71)$ & $1.77(1.51,2.73)$ \\
\hline ZA & $0.81(0.55,1.17)$ & $1.30(1.04,1.62)$ & $1.30(1.04,1.62)$ \\
\hline
\end{tabular}

The values in parentheses are the $95 \%$ confidence bands of the values of $\mathrm{d}$. In bold, the selected specification on the basis of the statistical significance of the deterministic terms. 